\title{
ON THE IMPROVEMENT OF ACCURACY IN INTEGRATION*
}

\author{
BY \\ MARK LOTKIN \\ Ballistic Research Laboratories, Aberdeen Proving Ground
}

1. Introduction. In solving numerically systems of ordinary differential equations in a stepwise manner it is frequently advisable, and certainly economically more efficient, to vary the step size as the integration proceeds. Further, when applying methods of integration that necessitate the computation at the "next" point of a number of approximate values for the unknown functions it may be desirable to employ the different approximations so as to increase the accuracy obtainable.

Both of these ideas are not new, and have been used on occasion previously, though mostly separately.

The scheme proposed here attempts to combine both techniques in a proper manner. The resulting methods are shown to work very effectively on a few critical examples; they are also in general sufficiently simple to be easily applicable to modern high speed computing machines.

2. The integration scheme. Let us assume that the integration problem is of the form

$$
y^{\prime}=f(x, y), \quad y\left(x_{0}\right)=y_{0},
$$

where $y$ and $f(x, y)$ are column matrices of $n$ components. The associated numerical problem is then of the form

$$
Y^{\prime}=F(x, Y),
$$

where $Y$ is a numerical approximation to $y$, and $F(x, Y)$ differs from $f(x, Y)$ by certain rounding errors which are due to the mode of construction of $f$ from its arguments $x, Y$. It will, in general, be convenient to denote mathematically exact quantities by small letters, their numerical representatives by corresponding capital letters. Concurrently, it should be recognized that the arithmetical operations involving such numerical approximants are of the "pseudo-arithmetical" type, of necessity introducing certain errors due to unavoidable rounding.

Let us suppose, further, that the solution of the associated numerical problem has proceeded through a sequence of points $x_{1}, x_{2}, \cdots, x_{k-1}$ to a point $x_{k}$, so that the last step size employed was $h=x_{k}-x_{k-1}$.

The integration scheme then consists simply in securing two approximations $V_{1}, V_{2}$ at $x_{k+1}=x_{k}+h$, and then utilizing their difference

$$
\delta Y=V_{1}-V_{2}
$$

for the double purpose of a) improving the accuracy of the $V_{1}$, and b) deciding whether or not a change in step size is warranted.

The importance of $\delta Y$ for accuracy considerations is easily recognized. Let the exact value of the solution be denoted by $y(x)$, so that

$$
Y_{k}=y\left(x_{k}\right)+\eta_{k}
$$

${ }^{*}$ Received March 23, 1954. 
$\eta_{k}$ represents the total error accumulated at $x_{k}$. The two approximants $V_{1}, V_{2}$ are now related to the exact value $y\left(x_{k+1}\right)$ as follows:

$$
V_{i}=y\left(x_{k+1}\right)+e_{i}, \quad i=1,2 .
$$

To fix the notation let $V_{2}$ denote the more exact determination of $y$ at $x_{k+1}$.

It may be assumed that the exact errors $e_{i}$ are composed linearly of terms due, respectively, to truncation, accumulation and rounding:

$$
e_{i}=\sum_{\alpha=1} b_{i \alpha} t_{\alpha}+\sum_{\beta-k, k-1} c_{i \beta} u_{\beta}+\sum_{\gamma=1} d_{i \gamma} r_{\gamma} .
$$

Since $\delta Y=e_{1}-e_{2}$ it is then clear that a relationship of the form

$$
e_{2}=a \delta Y+\sum_{\alpha} b_{\alpha}^{\prime} t_{\alpha}+\sum_{\beta} c_{\beta}^{\prime} u_{\beta}+\sum_{\gamma} d_{\gamma}^{\prime} r_{\gamma}
$$

must exist. The major part of $e_{2}$, which is easily computed numerically, is

$$
E_{2}=A \delta Y+\sum_{\alpha} B_{\alpha} T_{\alpha}+\sum_{\beta} C_{\beta} U_{\beta} .
$$

Let the difference between $e_{2}$ and $E_{2}$ be $\eta_{k+1}$. If we now take for the final value of $Y$ at $x_{k+1}$ the quantity

then obviously

$$
Y_{k+1}=V_{2}-E_{2}
$$

$$
Y_{k+1}=y\left(x_{k+1}\right)+\eta_{k+1} \text {. }
$$

The adjustment of step size may be tied to the magnitude of $E_{2}$, as follows. Suppose it is desired to keep $\left|e_{2}\right| \leq \epsilon$, where $\epsilon>0$ is prescribed. In general,

$$
\left|E_{2}\right|=m \epsilon \text {, }
$$

where $m$ is some positive number. It is then reasonable to expect these alternatives:

(a) if $m>1$ shorten $h$; (b) if $m<m_{0} \ll 1$ enlarge $h$; (c) if $m_{1}<m \leq 1$, leave $h$ unchanged.

Some of the methods for obtaining $\delta Y$ may be put conveniently into the following two categories:

I. Application of two distinct methods at full step. The "predictor-corrector" techniques [1] are of this type.

II. Application of a particular method first at full step, then at two successive half steps; these may be said to employ "local extrapolation to zero step" [2]. Special cases of each of these categories are considered below.

3. Predictor-corrector method. A good combination is provided by the choice of a low order central difference method for predictor, and Heun's method for corrector. The central difference formula is

$$
V_{1}=Y_{k-1}+2 h Y_{k}^{\prime}
$$

its truncation error is $t_{1}=\left(h^{3} / 3\right) y^{\prime \prime \prime}\left(s_{1}\right), x_{k-1}<s_{1}<x_{k+1}$. Heun's method, on the other hand, employs the formulas:

$$
\begin{aligned}
& V_{0}=Y_{k}+h Y_{k}^{\prime}, \\
& V_{2}=Y_{k}+(h / 2)\left(Y_{k}^{\prime}+V_{0}^{\prime}\right):
\end{aligned}
$$


the truncation error of Eq. (10), which is Euler's method, is $t_{0}=\left(h^{2} / 2\right) y^{\prime \prime}\left(s_{0}\right)$, that of Eq. (11) is $t_{2}=-\left(h^{3} / 12\right) y^{\prime \prime \prime}\left(s_{2}\right), x_{k}<s_{0}, s_{2}<x_{k+1}$. It is found that

$$
\begin{aligned}
& e_{1}=-t_{1}+2 p u_{k}+u_{k-1}+\cdots, \\
& e_{2}=-t_{2}-2^{-1} p t_{0}+\left(I+p+2^{-1} p^{2}\right) u_{k}+\cdots,
\end{aligned}
$$

where $p$ denotes the matrix of elements $p_{i j}=\partial f_{i} / \partial y_{i}, I$ is the unit matrix of $n$th order, and the omitted terms are due to local rounding only. Since $t_{1} \approx-4 t_{2}$, above equations result in

where

$$
E_{2}=\delta Y-\left(6 T_{2}+P T_{0}\right)+U_{k+1}
$$

$$
U_{k+1}=\left(2 I+P^{2}\right) U_{k}-U_{k-1},
$$

and the $T_{i}, P$ are evaluated at $x_{k+1}, V_{2}$.

Of interest may be the fact that by subtracting Eq. (13) from Eq. (12) there is obtained

$$
t_{2}=(1 / 5)\left[\delta Y-(1 / 2) p t_{0}+(I+p)^{-1} u_{k}-u_{k-1}\right]
$$

the resulting approximation

$$
t_{2} \approx \delta Y / 5
$$

was previously pointed out by Milne [1], p. 28 .

It is clear in general that combinations of formulas having "comparable" truncation errors $c_{i} h^{n} y^{(n)}\left(s_{i}\right)$ permit estimates similar to (16). However, combinations not possessing this property may still be useful.

Such a combination is, for example, that composed of formulas (10) and (11). In this case one gets

$$
\begin{aligned}
& e_{1}=-t_{0}+(I+p) u_{k}+\cdots, \\
& e_{2}=-t_{2}+\left(I+2^{-1} p\right) u_{k}+2^{-1} p e_{1}+\cdots .
\end{aligned}
$$

Replacing in the latter equation $e_{1}$ by $e_{2}+\delta Y$ there results

with

$$
E_{2}=(2 I-P)^{-1}\left[P \delta Y-2 T_{2}\right]+U_{k+1},
$$

$$
U_{k+1}=(2 I-P)^{-1}(2 I+P) U_{k} \text {. }
$$

Other predictor-corrector combinations may be analyzed in the same vein.

4. Local extrapolation. For purposes of illustration let us apply the technique of local extrapolation to Heun's method. For the first approximation, then, as by Eqs. (10) and (11),

$$
V_{1}=Y_{k}+(h / 2)\left(Y_{k}^{\prime}+V_{0}^{\prime}\right) \text {. }
$$

Next, let $\xi_{k}$ bisect the interval $\left\langle x_{k}, x_{k+1}\right\rangle$, and let us define $z_{k}=y\left(\xi_{k}\right), w=h / 2$. The second approximation $V_{2}$ is then obtained thus:

$$
\begin{aligned}
& W_{0}=Y_{k}+w Y_{k}^{\prime}, \\
& W_{1}=Y_{k}+(w / 2)\left(Y_{k}^{\prime}+W_{0}^{\prime}\right), \\
& W_{2}=W_{1}+w W_{1}^{\prime}, \\
& V_{2}=W_{1}+(w / 2)\left(W_{1}^{\prime}+W_{2}^{\prime}\right) .
\end{aligned}
$$


By Eq. (13), where $e_{2}$ now becomes $e_{1}$,

$$
e_{1}=-t_{2}-2^{-1} p t_{0}+\left(I+p+2^{-1} p^{2}\right) u_{k}+\cdots \text {. }
$$

Consequently,

$$
\begin{aligned}
e_{1}^{\prime} & \equiv W_{1}-y\left(\xi_{k}\right) \\
& =-\tau_{2}-2^{-1} \pi \tau_{1}+\left(I+\pi+2^{-1} \pi^{2}\right) u_{k}+\cdots,
\end{aligned}
$$

where the $\tau_{i}$ and $\eta$ differ from the $t_{i}$ and $p$ only in that $h$ is replaced by $w$. Since, therefore,

$$
\pi \approx 2^{-1} p, \quad \tau_{1} \approx 2^{-2} t_{1}, \quad \tau_{2} \approx 2^{-3} t_{2},
$$

it follows that

$$
8 e_{1}^{\prime} \approx e_{1}+\left(7 I+3 p+2^{-1} p^{2}\right) u_{k}+\cdots .
$$

On the other hand, a short calculation shows that

$$
e_{2}=\left(2 I+2^{-1} p+2^{-3} p^{2}\right) e_{1}^{\prime}-\left(I+2^{-1} p+2^{-3} p^{2}\right) u_{k}+\cdots .
$$

Therefore, using Eq. (20) while neglecting terms of order $p^{2}$, there is obtained

$$
E_{2}=(1 / 3)(I+P / 3) \delta Y+U_{k+1},
$$

where

$$
U_{k+1}=(I+P) u_{k} .
$$

It is not difficult to extend this discussion to more accurate integration procedures. . In doing this let us restrict ourselves to a single differential equation

$$
y^{\prime}=f(x, y), \quad y\left(x_{0}\right)=y_{0},
$$

and utilize the fact that most integration methods are equivalent to forms [3]

$$
\begin{gathered}
Y_{k}^{(i)}=F_{i}\left(x_{k}, Y_{k}, Y_{k}^{\prime}, \cdots Y_{k}^{(i-1)}\right), \quad i=1,2, \cdots N, \\
V_{0}=Y_{k}+h Y_{k}^{\prime}+\cdots+\left[h^{N-1} /(N-1) !\right] Y_{k}^{(N-1)}, \\
V_{1}=\sum_{i=0}^{\dot{1}} G_{0 i} Y_{k-i}+h\left(G_{1} V_{0}^{\prime}+\sum_{i=0}^{r} G_{1 i} Y_{k-i}^{\prime}\right)+\cdots \\
+h^{N}\left(G_{N} V_{0}^{(N)}+\sum_{i=0}^{r} G_{N i} Y_{k-i}^{(N)}\right) .
\end{gathered}
$$

Here the $g_{i j}$ are constants which in general depend on the range $r$ of points $x_{k-i}$ to the left of $x_{k}$, and $N$ indicates the degree of the polynomials for which the method is exact.

Proceeding in the same manner as described above one obtains eventually

$$
e_{2}=\delta Y \frac{1+g_{00}}{2^{N+1}-1-g_{00}}\left[1+p \frac{2^{N}\left(g_{1}+g_{10}\right)}{\left(2^{N+1}-1-g_{00}\right)\left(1+g_{00}\right)}\right]+\cdots .
$$


In those methods where $g_{00}=1$, such as Euler's, Heun's, Adam's, Gregory's, etc., Eq. (24) becomes

$$
e_{2}=\frac{\delta Y}{2^{N}-1}\left[1+p\left(g_{1}+g_{10}\right) \frac{2^{N-2}}{2^{N}-1}\right]+\cdots .
$$

In Heun's method, for example,

$$
\left(g_{00}, g_{1}, g_{10}\right)=(1,1 / 2,1 / 2), \quad N=2,
$$

and consequently

$$
E_{2}=(1 / 3)(1+P / 3) \delta Y+\cdots,
$$

which agrees with the principal part of Eq. (21).

5. Numerical example. The technique described above was tried out on the simple case of the differential equation

$$
y^{\prime}=y^{2},
$$

whose solution is $y(x)=(c-x)^{-1}$. For the integration the predictor-corrector combination of Euler-Heun was used:

$$
\begin{gathered}
V_{1}=Y_{k}+h Y_{k}^{\prime}, \quad V_{2}=Y_{k}+(h / 2)\left(Y_{k}^{\prime}+V_{1}^{\prime}\right), \\
E_{2}=(2-P)^{-1}\left(P \delta Y-2 T_{2}\right)+U_{k+1}, \quad U_{k+1}=(2-P)^{-1}(2+P) U_{k}, \\
Y_{k+1}=V_{2}-E_{2} .
\end{gathered}
$$

For our problem (26),

$$
P=2 h Y_{2}, \quad T_{2}=-\left(h^{3} / 2\right) Y_{2}^{4} .
$$

A. Initial Condition y $(0)=1$.

Starting with $y(0)=1$ it was desired to obtain the solution in the interval $0 \leq x<1$ to an accuracy of three decimal places.

The results of a number of integrations performed with Heun's method modified in a variety of ways are shown in Tables 1 and 2 .

TABLE 1

Improvement In Accuracy. Comparisons

(1)

(2)

(3)

(4)

(5)

\begin{tabular}{rcccccc}
$x$ & $Y$ (No Corr.) & Error & $Y$ (One Corr.) & Error & $Y$ (Two Corr.) & Error \\
\hline 0 & 1.000000 & $0.10^{-6}$ & 1.000000 & $0.10^{-6}$ & 1.000000 & $0.10^{-6}$ \\
.04 & 1.041632 & -35 & 1.041664 & -3 & 1.041667 & 0 \\
.08 & 1.086878 & -79 & 1.086950 & -7 & 1.086957 & 0 \\
.12 & 1.136229 & -135 & 1.136352 & -12 & 1.136363 & -1 \\
.16 & 1.190270 & -206 & 1.190458 & -18 & 1.190474 & -2 \\
.20 & 1.249702 & -298 & 1.249972 & -28 & 1.249996 & -4 \\
.24 & 1.315373 & -416 & 1.315749 & -40 & 1.315782 & -7 \\
.28 & 1.388318 & -571 & 1.388831 & -58 & 1.388875 & -14 \\
.32 & 1.469815 & -773 & 1.470506 & -83 & 1.470565 & -23
\end{tabular}


TABLE 2

Adjustment In Step Size

(1)

\begin{tabular}{lccc} 
& & $(2)$ & $(3)$ \\
$x$ & $Y$ & $\delta Y$ & $P$ \\
\hline 0 & 1.000000 & $0.10^{-6}$ & $8000.10^{-5}$ \\
.04 & 1.041667 & -1632 & 8333 \\
.32 & 1.470565 & -4406 & 17635 \\
.34 & 1.515129 & -1291 & 60604 \\
.36 & 1.562479 & -1413 & 62498 \\
.60 & 2.499982 & -5528 & 99994 \\
.61 & 2.564086 & -1582 & 51281 \\
.62 & 2.631564 & -1708 & 52631 \\
.76 & 4.166679 & -6528 & 83331 \\
.765 & 4.255334 & -1827 & 42553 \\
.770 & 4.347844 & -1947 & 43478 \\
.860 & 7.143004 & -8343 & 71429 \\
.8625 & 7.272882 & -2298 & 36364 \\
.8650 & 7.407570 & -2426 & 37038 \\
.9000 & 10.000355 & -5875 & 50001
\end{tabular}

(4)

(5)

$T_{2} \quad E_{2}$

$\left(-32.10^{-6}\right)$
-38
-150

$-21$

$-24$

$-156$

$-22$

$-24$

$-151$

$-20$

$-22$

$-163$

$-22$

$-24$

$-78$

$0.10^{-6}$
-35
-125
-22
-25
-140
-23
-25
-139
-22
-24
-154
-24
-25
-78

(6)

$E_{2}^{*} \quad$ Error

$\begin{array}{cc}0.10^{-6} & 0.10^{-6} \\ 114 & 0 \\ 343 & -23 \\ 66 & -23 \\ 469 & -21 \\ & -18\end{array}$

1. No corrections. For the accuracy required it is clearly sufficient to start with $h=0.04$. Using Heun's method without any modifications there are obtained the values listed in column (1) of Table 1; the errors, shown in column (2), are seen to exceed the tolerance $\epsilon=5.10^{-4}$ already at $x=0.28$.

2. One correction. Next the integration was carried out utilizing the expression for $E_{2}$ without the term $U_{k+1}$. The results are exhibited in column (3), the errors in column (4). This modification reduces the errors to about one tenth of their previous values.

3. Two corrections. The inclusion of $U_{k+1}$ in $E_{2}$ produced the results shown in column (5); the errors, in column (6), are reduced further considerably.

The continuation of column (5), together with the appropriate adjustment in step size, is shown in Table 2.

The integration interval was cut whenever the quantity

$$
E_{2}^{*}=(2-P)^{-1}\left[\left|P_{\delta} Y\right|+2\left|T_{2}\right|+(2+P)\left|U_{k}\right|\right]
$$

exceeded $5.10^{-4}$; this occurred at $x=0.36,0.62,0.77$ and 0.865 . At $x=0.9$ the step size was $h=0.0025$, the error $\eta=.000355$. Use of this step size from the beginning would require 360 lines of integration, while the computation shown in Table 2 necessitated only about one fifth of that number.

4. Use of $\delta Y$ for step adjustment. Instead of watching $E_{2}^{*}$ for adjusting the step size, the quantity $\delta Y$ could be used for this purpose, as suggested by Milne [1]. Cutting the interval whenever $\delta Y$ exceeded 0.0025 , which occurred at $x=0.20,0.48,0.67$, the error increased to .000496 already at $x=0.69$, so that the use of $E_{2}^{*}$ seemed superior, at least in this example.

5. The approximation $E_{2} \approx T_{2}$. Inspection of columns (4) and (5) shows that $E_{2} \approx T_{2}$, which is to be expected. If this assumption $E_{2} \approx T_{2}$ is introduced into Eq. (27), there results

$$
E_{2} \approx(4-P)^{-1} P \delta Y
$$


An integration based on this formula, with changes of interval whenever $|P \delta Y|<$ $5.10^{-4}$, produced an error of -0.000478 at $x=0.78$. A graphical illustration of the various techniques is also given in Fig. 1.

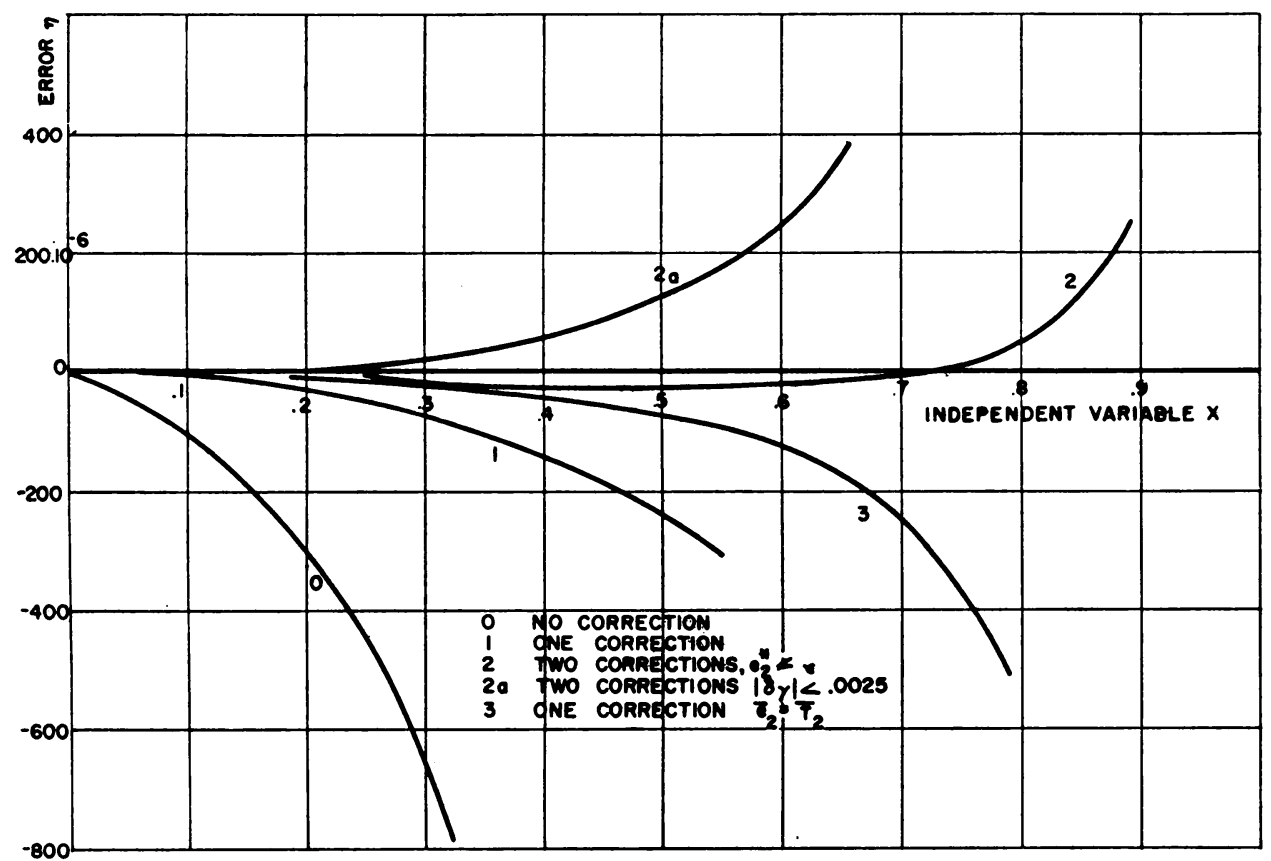

FIG. 1

Further inspection of Table 1 shows that the application of the unmodified Heun method leads to an error of $-693.10^{-6}$ at $x=0.60$. It is of interest to note that this error is quite closely approximated by the error propagation theorem derived in [3]. It was shown there that the total accumulated error for Heun's method is

$$
\Omega_{k} \approx\left[-1+2^{-1} p, h\right] \sum_{t=1}^{k} \lambda^{k-t}\left[\begin{array}{c}
-T_{t}+r_{1} \\
r_{2}
\end{array}\right],
$$

with

$$
\lambda=\Lambda / D=\left(g_{00}+p g_{10}\right) /\left(1-p g_{1}\right)=\left(1+2^{-1} p\right) /\left(1-2^{-1} p\right) .
$$

In our example $r_{1}, r_{2} \approx 5.10^{-6}$, so that the contributions due to these errors may be neglected. Consequently,

$$
\Omega_{k} \approx \frac{2-p}{2}\left(\frac{2+p}{2-p}\right)^{k} \sum_{i=1}^{k}\left(\frac{2-p}{2+p}\right)^{i} T_{t} .
$$

Using in (31) the average value $2^{-1}\left(p_{1}+p_{k}\right)$ for $p$, there is obtained for the total accumulated error at $x=0.60$ the value $-723.10^{-6}$, which differs from the exact error $-693.10^{-6}$ by only about $4 \%$. 
B. Initial Condition $y(2)=-1$. The purpose of this computation is to illustrate the possibility of continual enlargement of $h$ without the loss of accuracy. The results are shown in Table 3. First Heun's method without any modification was applied, at a

TABLE 3

Adjustment In Step Size

(1)

$\begin{array}{rcc}x & Y \text { (No Corr.) } & \text { Error } \\ 2.00 & -1.000000 & 0.10^{-6} \\ 2.04 & -.961568 & -30 \\ 2.44 & -.694567 & -123 \\ 2.52 & -.658018 & -123 \\ 2.60 & -.625121 & -121 \\ 3.40 & -.416751 & -84\end{array}$

(3)

$\begin{array}{cc}Y \text { (Two Corr.) } & T_{2} \\ -1.000000 & \left(-32.10^{-0}\right) \\ -.961538 & -24 \\ -.694447 & -7 \\ -.657893 & -48 \\ -.625996 & -39 \\ -.416664 & -8 \\ -.390618 & -48 \\ -.367638 & -37 \\ -.240379 & -7 \\ -.223205 & -41 \\ -.208323 & -31 \\ -.148801 & -8\end{array}$

(5)

$E_{2}$
$0.10^{-6}$
-30
-7
-57
-46
-8
-59
-45
-7
-51
-38
-9

(6)

\begin{tabular}{cc}
\multicolumn{1}{c}{$E_{2}^{*}$} & Error \\
$0.10^{-6}$ & $0.10^{-6}$ \\
86 & 0 \\
25 & -3 \\
175 & 2 \\
& 4 \\
25 & 3 \\
1777 & 7 \\
& 9 \\
25 & 6 \\
140 & 9 \\
& 10 \\
27 & 9
\end{tabular}

constant step of $h=0.04$. The errors are listed in column (2); since now $p<0$ the errors tend to damp out.

In the remaining columns (3) to (7) there are again exhibited the computations using the technique described in Eqs. (27). The interval was doubled whenever $E_{2}^{*}$ fell below $25.10^{-6}$, which occurred at $x=2.48,3.48,5.32,8.04, \cdots$. The error at $x=7.72$ was $9.10^{-6}$, which could hardly have been improved upon even with a constant step size of $h=0.04$, at a computational labor of 143 lines of integration instead of the 42 needed for Table 3.

\section{REFERENCES}

[1] W. E. Milne, Numerical solution of differential equations, Wiley \& Sons, New York, 1953

[2] L. F. Richardson, The deferred approach to the limit, Phil. Trans. 226, 299-349 (1927)

[3] M. Lotkin, The propagation of error in numerical integrations, BRL Report No. 875, 34 pp., 1953 